\title{
A production inventory model with flexible manufacturing, random machine breakdown and stochastic repair time
}

\author{
S.R. Singh ${ }^{\mathrm{a}}$ and Leena Prasher ${ }^{\mathrm{b}}$
}

${ }^{a}$ Department of Mathematics, D. N. College, Meerut, 250103,India

${ }^{b}$ Centre for Mathematical Sciences, Banasthali University, Banasthali, Rajasthan 304022, India

CHR ON I C LE ABSTRACT

Article history:

Received January 262014

Received in Revised Format

July 52014

Accepted July 52014

Available online

July 122014

Keywords:

Volume flexible manufacturing

system

Stochastic machine breakdown

Stochastic repair time

Stock dependent demand

\begin{abstract}
This paper derives a production inventory model over infinite planning horizon with flexible but unreliable manufacturing process and the stochastic repair time. Demand is stock dependent and during the period of sale it depends on reduction on selling price. Production rate is a function of demand and reliability of the production equipment is assumed to be exponentially decreasing function of time. Repair time is estimated using uniform probability density function. The objective of the study is to determine the optimal policy for production system, which maximizes the total profit subject to some constraints under consideration. The results are discussed with a numerical example to illustrate the theory.
\end{abstract}

\section{Introduction}

Classical economic production inventory model assumes that manufacturing systems are perfectly reliable. This assumption, however, does not hold for many real systems. Even the best and the most modern production systems face the situation of sudden machine breakdown, and the time taken in the repair of machine also sometimes depend on the type of injury occurred. Reliability of the production equipment is a crucial factor for keeping the synchronization in the production system, and may harm the organization if the existing uncertainty of the production equipment is not taken into account and is planned, accordingly. In this study, the production system is taken as flexible to produce as per the demand but is not reliable. The production equipment may breakdown at any random time and the repair time is also assumed to be stochastic in nature. During a production run, it may shift from incontrol state to out-of control state, and the production process may have to be stopped at any random time. The objective of this study is to determine the expected optimal production run time with a view to maximizing the expected profit per unit time.

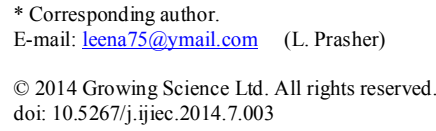




\section{Literature Review}

The classical production inventory model assumes that manufacturing system is perfectly reliable. Such an assumption appears impractical in real system. Researchers, therefore, have been attracted towards machine breakdown effects on production inventory problem. The effects of machine breakdown and corrective maintenance were studied by Groenevelt et al. (1992). They studied two production control policies to deal with stochastic machine breakdowns. The first one assumes that the production of the interrupted lot is not resumed after a breakdown. While the second policy considers that the production of the interrupted lot will be immediately resumed after the breakdown if the current on-hand inventory level is below a certain threshold level. Incorporated preventive maintenance to production inventory model was done by Cheung and Hausman (1997). They developed a mathematical model with random machine breakdowns and considered preventive maintenance and safety stock. Wang (2004) developed an EPQ mathematical model where production shifts from an in-control state to an out-of control state with a general shift distribution.

Giri et al. (2005) developed EMQ model with machine failure and general repair time. They proposed a model to determine the production rate and production lot size to minimize the expected total cost. Giri and Dohi (2005) developed EMQ model with random variables, corrective and preventive repair. They proposed solution procedure and computational algorithms to find the optimal production rate and lot size. Lin and Gong (2006) developed EPQ model deteriorating inventory model with machine breakdown and fix repair time. Chiu et al. (2007) derived an economic production quantity (EPQ) model with scrap, rework, and stochastic machine breakdowns, assuming some portion of the defective items to be scrapped and the other part to be repairable. Leung (2007) derived a generalized geometric programming solution to an EPQ model with flexibility and reliability considerations. Chakraborty et a,. (2008) developed an EPQ model considering production system that may shift from in-control state to out-of control state or may breakdown at any random time during a production period. Ferik (2008) developed an EPQ model for unreliable manufacturing facility. Similar research for EPQ model with imperfect process has been done by Liao et al. (2009). Singh and Singh (2010) worked on supply chain model with stochastic lead time under imprecise partially backlogging for expiring items. Widyadana and Wee (2011) developed production inventory model with random machine breakdown and stochastic repair time. They proved that stochastic repair model tends to have larger optimal cost than fixed repair time model.

An increase in the shelf space can influence more customers. In this connection, the observations made by Levin et al. (1972) and Silver and Peterson (1985) was worth noting, that the presence of greater quantity of the same item tends to attract more customers. The reason behind this fact is a typical psychology of the customers. They may have the feeling of obtaining a wide range for selection when a large amount is stored/displayed. Gupta and Vrat (1986) developed models for stock dependent consumption rate. Mandal and Phaujdar (1989) developed an inventory model for deteriorating items and stock dependent consumption rate. Schweitzer and Seidmann (1991) established optimizing processing rate for flexible manufacturing systems. Giri and Chaudhuri (1998) developed deterministic model of perishable inventory with stock-dependent demand rate and nonlinear holding cost and proved that the nonlinear holding cost affected the total average cost. Sana et al. (2006) established a production-inventory model for a deteriorating item with trended demand and shortages. Teng and Chang (2005) proposed economic production model for deteriorating item with price and stock dependent demand. Singh and Jain (2009) worked on reserve money for an EOQ model in an inflationary environment under supplier credits. Singh (2010) gave an inventory model for deteriorating items with shortages and stock-dependent demand under inflation for two-shops under one management. Yadav et al. (2012) developed an inventory model of deteriorating items with stock dependent demand using genetic algorithm in fuzzy environment. Dem and Singh (2012) investigated an EPQ model for damageable items with multivariate demand and flexible manufacturing. Dem and Singh (2013) developed a production model for imperfect production process under volume flexibility. 
Goyal et al. (2013) explored an inventory system with variable demand as well as production under partially backordered shortages.

\section{Assumptions and Notations}

The following assumptions and notations are used throughout the model.

\subsection{Assumptions}

1. The production rate is a function of demand $P=\ell D(q), \ell>1$

2. The demand rate $D(q)$ is a function of on hand inventory level in the interval $(0, \mu)$ and is given by $D(q)=\alpha+\beta q, 0<\beta<1, q \geq 0$ where $\beta$ denotes the shape parameter and is a measure of responsiveness of the demand to changes in the level of on hand inventory and $\alpha$ denotes the scale parameter.

3. After $t=\mu$, the sale starts and demands rate $D(q)=(\alpha+\beta q)\left(a b^{r}\right), 0 \leq r \leq 1$ is taken as function of stock displayed and reduction on the selling price.

4. The time horizon of the inventory system is infinite. Only a typical planning schedule of expected length $E(T)$ is considered, all remaining cycles are identical.

5. Machine repair time is independent of machine breakdown.

\subsection{Notations}

$q(t)$ : On hand inventory level of products.

$D(q)$ : Demand rate,

$P$ : Production rate $P=\ell D(q)$ where $\ell$ is a scale parameter, $P>D(q), \ell>1$

$K \quad:$ Set up cost

$S \quad$ : Selling price per item

$r \quad$ : Reduction (in \%) of selling price of products

$h$ : Holding cost per unit of item per unit time

$T_{1} \quad$ : Time when production stops

$T_{p} \quad$ : Time when machine breakdown occurs

$\mu \quad$ : Time when sale of products starts

$T_{2} \quad$ : Time when inventory of products vanishes and shortages start to accumulate which causes lost sales

$E(T) \quad$ : Expected Duration of a production cycle

$E(P C) \quad$ : Expected production cost

$E(H C)$ : Expected holding cost in the production cycle

$E(S R) \quad$ : Expected sales revenues from items in the production cycle

$E(L S C):$ Expected lost sales cost

$E(T A P):$ Expected total profit per unit time from the production cycle

\section{Formulation of model}

We consider a system in which the manufacturing process is flexible as long as machine is working efficiently and hence can produce as per the demand rate. Generally, reliability of the machine is assumed to be an exponentially decreasing function of time and therefore the probability density 
function for machine breakdown is assumed as $\mathrm{f}\left(\mathrm{T}_{\mathrm{p}}\right)=\lambda \mathrm{e}^{-\lambda \mathrm{T}_{\mathrm{p}}}$. The demand function for the products is assumed as stock dependent, which is $D(q)=\alpha+\beta q$ where $\beta$ denotes the shape parameter and is a measure of responsiveness of the demand to changes in the level of on hand inventory and $\alpha$ is the deterministic factor. Many of the organizations decide to sell their goods at reduced prices after predestined time, which is normally known as sale period. The demand function during the sale period is assumed as function of stock displayed as well as discounted price for items and so the demand function during sale period is assumed as $(\alpha+\beta \mathrm{q})\left(\mathrm{ab}^{\mathrm{r}}\right), a>0, b>1,0 \leq r \leq 1$, where $r$ is the reduction in the selling price, Geometrical description is shown in Fig. 1.

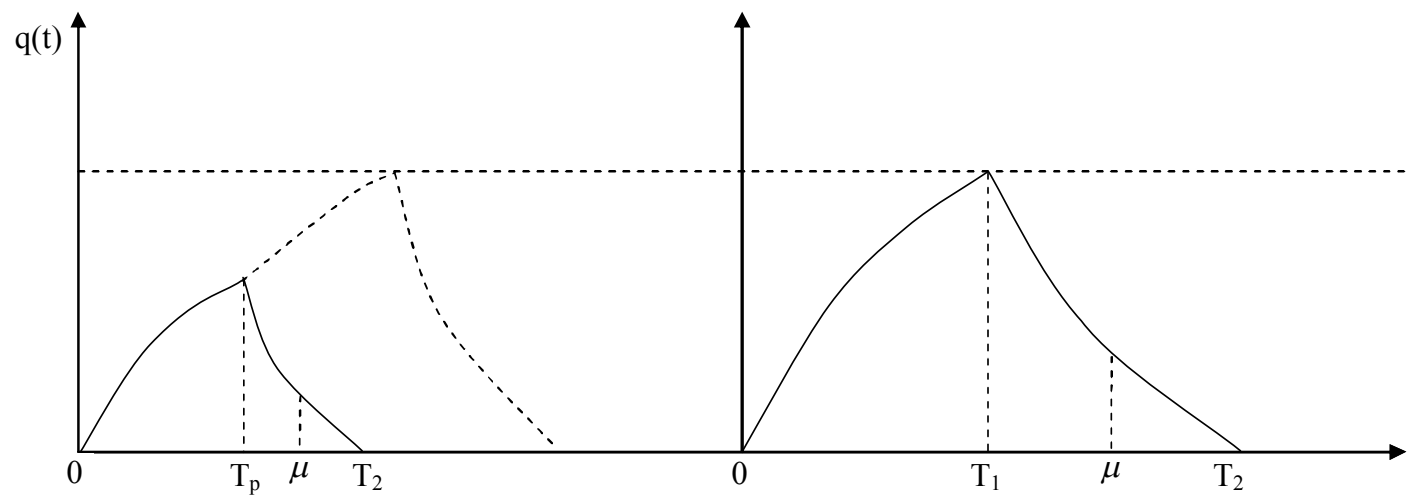

Fig. 1. Production system with lost sales.

The production cycle begins with zero inventory and starts at $t=0$. As time advances, if the machine does not breakdown in the production period $\left[0, T_{1}\right]$ inventory level of products pile up even after meeting market demand in the interval $0 \leq t \leq T_{1}$, as shown by Eq. (1). Feasibility of this assumption implies $\ell-1$ must be greater than zero. Production is stopped at time $t=T_{1}$ and the

inventory level of products decreases due to demand in the interval $\left[T_{1}, \mu\right]$ as depicted by Eq (2). The sale period starts at $t=\mu$ and the inventory of products further decreases due to demand and reaches zero at time $t=T_{2}$ as shown by Eq.(3). Since the machine has a possibility of breakdown, the machine may not work the whole $T_{1}$ period. When the machine breakdown occurs, the production period stops at $t=T_{p}$ and machine requires repair time. As repair time is also stochastic, production may not always be possible and lost sales may occur.

Case I: $\quad$ When $\mu$ is less than the cycle length

$$
\begin{aligned}
& \frac{\mathrm{dq}}{\mathrm{dt}}=\mathrm{P}-\mathrm{D}(\mathrm{q}), \quad q(0)=0, \quad 0 \leq \mathrm{t} \leq \mathrm{T}_{1} \\
& \frac{\mathrm{dq}}{\mathrm{dt}}=-(\alpha+\beta \mathrm{q}), \quad q\left(T_{1}^{+}\right)=q\left(T_{1}^{-}\right), \quad \mathrm{T}_{1} \leq \mathrm{t} \leq \mu \\
& \frac{\mathrm{dq}}{\mathrm{dt}}=-(\alpha+\beta \mathrm{q})\left(\mathrm{ab}^{\mathrm{r}}\right), \quad q\left(T_{2}\right)=0, \quad \mu \leq \mathrm{t} \leq \mathrm{T}_{2}
\end{aligned}
$$

Solving Eq. (1), Eq. (2) and Eq. (3) using appropriate boundary conditions, the inventory levels in various intervals are calculated as below 
$\mathrm{q}(\mathrm{t})=\frac{\alpha\left(\mathrm{e}^{(\ell-1) \beta \mathrm{t}}-1\right)}{\beta}, \quad 0 \leq \mathrm{t} \leq \mathrm{T}_{1}$

$\mathrm{q}(\mathrm{t})=\frac{\alpha}{\beta}\left(\mathrm{e}^{\left(\ell \mathrm{T}_{1}-\mathrm{t}\right) \beta}-1\right), \mathrm{T}_{1} \leq \mathrm{t} \leq \mu$

$\mathrm{q}(\mathrm{t})=\frac{\alpha}{\beta}\left(\mathrm{e}^{\mathrm{ab}^{\mathrm{r}}\left(\mathrm{T}_{2}-\mathrm{t}\right) \beta}-1\right), \quad \mu \leq \mathrm{t} \leq \mathrm{T}_{2}$

To find the relation between the variables using the Taylor series expansion of solutions and using continuity condition, we have $\mathrm{T}_{2}=\frac{\mu\left(\mathrm{ab}^{\mathrm{r}}-1\right)+\ell \mathrm{T}_{1}}{\mathrm{ab}^{\mathrm{r}}}$.

For the feasibility of practical situation assumed in case I, $T_{2}$ must be greater than $\mu$ which in turn implies $\ell T_{1}-\mu>0$. Total Inventory in the complete production cycle is calculated as below

$$
\begin{aligned}
& \int_{0}^{\mathrm{T}_{1}} \frac{\alpha}{\beta}\left(\mathrm{e}^{(\ell-1) \beta \mathrm{t}}-1\right) \mathrm{dt}+\frac{\alpha}{\beta} \int_{\mathrm{T}_{1}}^{\mu}\left(\mathrm{e}^{\left(\ell \mathrm{T}_{1}-\mathrm{t}\right) \beta}-1\right) \mathrm{dt}+\int_{\mu}^{\mathrm{T}_{2}} \frac{\alpha}{\beta}\left(\mathrm{e}^{\mathrm{ab}\left(\mathrm{T}_{2}-\mathrm{t}\right) \beta}-1\right) \mathrm{dt} \\
& =\frac{\alpha}{\beta}\left[\frac{\ell \mathrm{e}^{(\ell-1) \mathrm{T}_{1} \beta}-1}{(\ell-1) \beta}+\frac{\left(1-\mathrm{ab}^{\mathrm{r}}\right) \mathrm{e}^{\left(\ell \mathrm{T}_{1}-\mu\right) \beta}-1}{\left(\mathrm{ab}^{\mathrm{r}} \beta\right)}-\frac{\left(\mu\left(\mathrm{ab}^{\mathrm{r}}-1\right)+\ell \mathrm{T}_{1}\right)}{\mathrm{ab}^{\mathrm{r}}}\right]
\end{aligned}
$$

If machine breakdown occurs at $t=T_{p}$, then (4) is formulated as below

$$
\mathrm{E}(\mathrm{I})=\left[\begin{array}{l}
\frac{\alpha}{\beta}\left[\frac{\ell \mathrm{e}^{(\ell-1) \mathrm{T}_{\mathrm{p}} \beta}-1}{(\ell-1) \beta}+\frac{\left(1-\mathrm{ab}^{\mathrm{r}}\right) \mathrm{e}^{\left(\ell \mathrm{T}_{\mathrm{p}}-\mu\right) \beta}-1}{\left(\mathrm{ab}^{\mathrm{r}}\right) \beta}-\frac{\mu\left(\mathrm{ab}^{\mathrm{r}}-1\right)+\ell \mathrm{T}_{\mathrm{p}}}{\mathrm{ab}^{\mathrm{r}}}\right] \ldots \ldots . . . \ldots . . \mathrm{T}_{\mathrm{p}}<\mathrm{T}_{1} \\
\frac{\alpha}{\beta}\left[\frac{\ell \mathrm{e}^{(\ell-1) \mathrm{T}_{\mathrm{1}} \beta}-1}{(\ell-1) \beta}+\frac{\left(1-\mathrm{ab}^{\mathrm{r}}\right) \mathrm{e}^{\left(\ell \mathrm{T}_{1}-\mu\right) \beta}-1}{\left(\mathrm{ab}^{\mathrm{r}}\right) \beta}-\frac{\mu\left(\mathrm{ab}^{\mathrm{r}}-1\right)+\ell \mathrm{T}_{1}}{\mathrm{ab}^{\mathrm{r}}}\right] \ldots \ldots . . . \ldots . . \mathrm{T}_{\mathrm{p}}>\mathrm{T}_{1}
\end{array}\right]
$$

Using the machine breakdown probability density function of $T_{p}, \mathrm{f}\left(\mathrm{T}_{\mathrm{p}}\right)=\lambda \mathrm{e}^{-\lambda \mathrm{T}_{\mathrm{p}}}, T_{p}>0$, the expected inventory is calculated as:

$$
\begin{aligned}
& \int_{\mathrm{T}_{\mathrm{p}}=0}^{\mathrm{T}_{\mathrm{p}}=\mathrm{T}_{\mathrm{l}}} \mathrm{E}(\mathrm{I}) \lambda \mathrm{e}^{-\lambda \mathrm{T}_{\mathrm{p}}} \mathrm{d} \mathrm{T}_{\mathrm{p}}++\int_{\mathrm{T}_{\mathrm{p}}=\mathrm{T}_{1}}^{\infty} \mathrm{E}(\mathrm{I}) \lambda \mathrm{e}^{-\lambda \mathrm{T}_{\mathrm{p}}} \mathrm{d} \mathrm{T}_{\mathrm{p}} \\
& \int_{\mathrm{T}_{\mathrm{p}}=0}^{\mathrm{T}_{\mathrm{p}}=\mathrm{T}_{\mathrm{T}}} \frac{\alpha}{\beta}\left[\frac{\ell \mathrm{e}^{(\ell-1) \mathrm{T}_{\mathrm{p}} \beta}-1}{(\ell-1) \beta}+\frac{\left(1-\mathrm{ab}^{\mathrm{r}}\right) \mathrm{e}^{\left(\ell \mathrm{T}_{\mathrm{p}}-\mu\right) \beta}-1}{\left(\mathrm{ab}^{\mathrm{r}}\right) \beta}-\frac{\mu\left(\mathrm{ab}^{\mathrm{r}}-1\right)+\ell \mathrm{T}_{\mathrm{p}}}{\mathrm{ab}^{\mathrm{r}}}\right] \lambda \mathrm{e}^{-\lambda \mathrm{T}_{\mathrm{p}}} \mathrm{dT}_{\mathrm{p}}+\int_{\mathrm{T}_{\mathrm{p}}=\mathrm{T}_{\mathrm{I}}}^{\infty} \mathrm{E}(\mathrm{I}) \lambda \mathrm{e}^{-\lambda \mathrm{T}_{\mathrm{p}}} \mathrm{dT}_{\mathrm{p}} \\
& =\frac{\lambda \alpha}{\beta}\left[\frac{\ell\left(\mathrm{e}^{((\ell-1) \beta-\lambda) \mathrm{T}_{1}}-1\right)}{(\ell-1) \beta((\ell-1) \beta-\lambda)}+\frac{\mathrm{e}^{-\lambda \mathrm{T}_{1}}-1}{\lambda(\ell-1) \beta}+\frac{\mathrm{e}^{-\lambda \mathrm{T}_{1}}-1}{\left(\mathrm{ab}^{\mathrm{r}}\right) \beta \lambda}+\frac{\left(1-\mathrm{ab}^{\mathrm{r}}\right)\left(\mathrm{e}^{(\ell \beta-\lambda) \mathrm{T}_{1}-\mu \beta}-\mathrm{e}^{-\mu \beta}\right)}{\left(\mathrm{ab}^{\mathrm{r}}\right) \beta(\ell \beta-\lambda)}+\right.
\end{aligned}
$$




$$
\begin{aligned}
& \left.\frac{\mu\left(\mathrm{ab}^{\mathrm{r}}-1\right)\left(\mathrm{e}^{-\lambda \mathrm{T}_{1}}-1\right)}{\left(\mathrm{ab}^{\mathrm{r}}\right) \lambda}-\frac{\ell}{\mathrm{ab}^{\mathrm{r}}}\left(\frac{-\mathrm{T}_{1} \mathrm{e}^{-\lambda \mathrm{T}_{1}}}{\lambda}+\frac{1-\mathrm{e}^{-\lambda \mathrm{T}_{1}}}{\lambda^{2}}\right)\right] \\
& +\frac{\alpha}{\beta}\left[\frac{\ell \mathrm{e}^{(\ell-1) \mathrm{T}_{1} \beta}-1}{(\ell-1) \beta}+\frac{\left(1-\mathrm{ab}^{\mathrm{r}}\right)\left(\mathrm{e}^{\left(\ell \mathrm{T}_{1}-\mu\right) \beta}\right)-1}{\left(\mathrm{ab}^{\mathrm{r}}\right) \beta}-\frac{\mu\left(\mathrm{ab}^{\mathrm{r}}-1\right)+\ell \mathrm{T}_{1}}{\mathrm{ab}^{\mathrm{r}}}\right] \mathrm{e}^{-\lambda \mathrm{T}_{1}}
\end{aligned}
$$

Expected holding cost in complete Production cycle, E (HC)

$$
\begin{aligned}
& =\mathrm{h}\left[\frac{\alpha \ell \mathrm{e}^{((\ell-1) \beta-\lambda) \mathrm{T}_{\mathrm{I}}}}{\beta((\ell-1) \beta-\lambda)}-\frac{\lambda \alpha \ell}{(\ell-1) \beta^{2}((\ell-1) \beta-\lambda)}+\frac{\ell \alpha\left(1-\mathrm{ab}^{\mathrm{r}}\right) \mathrm{e}^{(\ell \beta-\lambda) \mathrm{T}_{1}-\mu \beta}}{\left(\mathrm{ab}^{\mathrm{r}}\right) \beta(\ell \beta-\lambda)}-\frac{\left(1-\mathrm{ab}^{\mathrm{r}}\right) \mathrm{e}^{-\mu \beta} \lambda \alpha}{\left(\mathrm{ab}^{\mathrm{r}}\right) \beta^{2}(\ell \beta-\lambda)}\right. \\
& \left.-\frac{\alpha}{\beta^{2}}\left(\frac{1}{\ell-1}+\frac{1}{\left(\mathrm{ab}^{\mathrm{r}}\right)}\right)-\frac{\mu\left(\mathrm{ab}^{\mathrm{r}}-1\right) \alpha}{\left(\mathrm{ab}^{\mathrm{r}}\right) \beta}-\frac{\ell \alpha}{\left(\mathrm{ab}^{\mathrm{r}}\right) \beta}\left(\frac{1-\mathrm{e}^{-\lambda \mathrm{T}_{1}}}{\lambda}\right)\right]
\end{aligned}
$$

Lost sales occur when repair time exceeds $T_{2}$. Assuming that machine repair time $t$ is a random variable and is uniformly distributed over the interval $[0, c]$. The probability density function $\mathrm{f}(\mathrm{t})$ for the repair time is given by $f(t)= \begin{cases}\frac{1}{c}, & 0 \leq t \leq c \\ 0, & \text { otherwise }\end{cases}$

For feasibility of the practical situation considered in the model, $T_{2}$ must be less than c, otherwise there will be no lost sales interval as lost sales occur when repair time exceeds $T_{2}$. Substituting the uniform probability density function of repair time and machine breakdown probability density function, expected lost sales cost is obtained as below

Expected Lost sales cost,

$\mathrm{E}(\mathrm{LSC})=\frac{\mathrm{S} \alpha}{\mathrm{c}} \int_{\mathrm{T}_{\mathrm{p}}=0}^{\mathrm{T}_{1}} \int_{\mathrm{t}=\mathrm{T}_{2}}^{\mathrm{c}}\left(\mathrm{t}-\mathrm{T}_{2}\right) \lambda \mathrm{e}^{-\lambda \mathrm{T}_{\mathrm{p}}} \mathrm{dtdT}_{\mathrm{p}}=\frac{\lambda \mathrm{S} \alpha}{2 \mathrm{c}} \int_{0}^{\mathrm{T}_{1}}\left(\mathrm{c}-\frac{\mu\left(a b^{\mathrm{r}}-1\right)+\ell \mathrm{T}_{\mathrm{p}}}{\mathrm{ab} b^{\mathrm{r}}}\right)^{2} \mathrm{e}^{-\lambda \mathrm{T}_{\mathrm{p}}} \mathrm{dT} \mathrm{T}_{\mathrm{p}}$

$$
\frac{\lambda \mathrm{S} \alpha}{2 \mathrm{c}}\left[\frac{\mathrm{A}^{2}\left(1-\mathrm{e}^{-\lambda \mathrm{T}_{1}}\right)}{\lambda}+\frac{\ell^{2}}{\left(\mathrm{ab}^{\mathrm{r}}\right)^{2}}\left(\frac{-\mathrm{T}_{1}^{2} \mathrm{e}^{-\lambda \mathrm{T}_{1}}}{\lambda}-\frac{2 \mathrm{~T}_{1} \mathrm{e}^{-\lambda \mathrm{T}_{1}}}{\lambda^{2}}+\frac{2\left(1-\mathrm{e}^{-\lambda \mathrm{T}_{1}}\right)}{\lambda^{3}}\right)-\frac{2 \mathrm{~A} \ell}{\mathrm{ab}^{\mathrm{r}}}\left(\frac{-\mathrm{T}_{1} \mathrm{e}^{-\lambda \mathrm{T}_{1}}}{\lambda}+\frac{1-\mathrm{e}^{-\lambda \mathrm{T}_{1}}}{\lambda^{2}}\right)\right]
$$

where $\mathrm{A}=\mathrm{c}-\frac{\mu\left(a \mathrm{~b}^{\mathrm{r}}-1\right)}{\mathrm{ab}}$

Next, we calculate production cost as below

$C_{p} \int_{0}^{T_{1}} P d t=C_{p} \int_{0}^{T_{1}} l D(q) d t=C_{p} l \alpha\left(\frac{e^{(l-1) \beta T_{1}}-1}{(l-1) \beta}\right)$

When machine breakdown occurs at $t=T_{p}$, then Eq. (7) can be formulated as

$\mathrm{PC}= \begin{cases}C_{p} l \alpha\left(\frac{e^{(l-1) \beta T_{p}}-1}{(l-1) \beta}\right) & \text { If } \ldots . . T_{p}<T_{1} \\ C_{p} l \alpha\left(\frac{e^{(l-1) \beta T_{1}}-1}{(l-1) \beta}\right) & \text { If } \ldots . . T_{p}>T_{1}\end{cases}$ 
Using the above formulation, expected production cost can be obtained as

$$
\begin{aligned}
& E(P C)=\int_{T_{p}=0}^{T_{p}=T_{1}} C_{p} l \alpha\left(\frac{e^{(l-1) \beta T_{p}}-1}{(l-1) \beta}\right) \lambda e^{-\lambda T_{p}} d T_{p}+\int_{T_{p}=T_{1}}^{T_{p}=\infty} C_{p} l \alpha\left(\frac{e^{(l-1) \beta T_{1}}-1}{(l-1) \beta}\right) \lambda e^{-\lambda T_{p}} d T_{p} \\
& =C_{p} l \alpha\left(\frac{e^{((l-1) \beta-\lambda) T_{1}}-1}{(l-1) \beta-\lambda}\right)
\end{aligned}
$$

The expected total inventory cost consists of set up cost, expected holding cost, expected lost sales cost and expected production cost.

Expected total cost, $E(T C)=K+E(H C)+E(L S C)+E(P C)$

Next we calculate sales revenue in the complete production cycle,

$$
=S\left(\int_{0}^{T_{1}}(\alpha+\beta q) d t+\int_{T_{1}}^{\mu}(\alpha+\beta q) d t\right)+S(1-r)\left(\int_{\mu}^{T_{2}}(\alpha+\beta q)\left(a b^{r}\right) d t\right)=S \alpha\left(\frac{e^{(\ell-1) \beta T_{1}}-1}{(\ell-1) \beta}+\frac{r\left(1-e^{\left(\ell T_{1}-\mu\right) \beta}\right)}{\beta}\right)
$$

If machine break down occurs at $t=T_{p}$, then sales revenues are formulated as

$$
\mathrm{SR}=\left[\begin{array}{l}
\mathrm{S} \alpha\left(\frac{\mathrm{e}^{(\ell-1) \beta \mathrm{T}_{\mathrm{p}}}-1}{(\ell-1) \beta}+\frac{\mathrm{r}\left(1-\mathrm{e}^{\left(\ell \mathrm{T}_{\mathrm{p}}-\mu\right) \beta}\right)}{\beta}\right) \ldots . . . . . . . \mathrm{T}_{\mathrm{p}}<\mathrm{T}_{1} \\
\mathrm{~S} \alpha\left(\frac{\mathrm{e}^{(\ell-1) \beta \mathrm{T}_{1}}-1}{(\ell-1) \beta}+\frac{\mathrm{r}\left(1-\mathrm{e}^{\left(\ell \mathrm{T}_{1}-\mu\right) \beta}\right)}{\beta}\right) \ldots . . . . . . \mathrm{T}_{\mathrm{p}}>\mathrm{T}_{1}
\end{array}\right]
$$

Using the probability density function of machine breakdown time $T_{p}$, the expected sales revenues of complete production cycle are obtained as

$$
\begin{aligned}
& E(S R)=\int_{0}^{T_{1}} S \alpha\left(\frac{e^{(\ell-1) \beta T_{p}}-1}{(\ell-1) \beta}+\frac{r\left(1-e^{\left(\ell T_{p}-\mu\right) \beta}\right)}{\beta}\right) \lambda e^{-\lambda T_{p}} d T_{p}+\int_{T_{1}}^{\infty} S \alpha\left(\frac{e^{(\ell-1) \beta T_{1}}-1}{(\ell-1) \beta}+\frac{r\left(1-e^{\left(\ell T_{1}-\mu\right) \beta}\right)}{\beta}\right) \lambda e^{-\lambda T_{p}} d T_{p} \\
& =\frac{S \alpha \ell\left(e^{((\ell-1) \beta-\lambda) T_{1}}-1\right)}{(\ell-1) \beta-\lambda}+\frac{S \alpha r \lambda e^{-\mu \beta}}{(\ell \beta-\lambda) \beta}-\frac{S \ell \alpha r e^{(\ell \beta-\lambda) T_{1}-\mu \beta}}{(\ell \beta-\lambda)}+\frac{S \alpha r}{\beta}
\end{aligned}
$$

The expected total replenishment time is the sum of expected production up time period, non production period and expected repair time after $t=T_{2}$. Therefore, Expected total time, $\mathrm{E}(\mathrm{T})=\mathrm{E}\left(\mathrm{T}_{2}\right)+$ Expected Repair time

$$
\begin{aligned}
& \int_{T_{\mathrm{p}}=0}^{\mathrm{T}_{1}} \mathrm{~T}_{2} \lambda \mathrm{e}^{-\lambda \mathrm{T}_{\mathrm{p}}} \mathrm{dT} \mathrm{T}_{\mathrm{p}}+\int_{\mathrm{T}_{\mathrm{p}}=\mathrm{T}_{1}}^{\infty} \mathrm{T}_{2} \lambda \mathrm{e}^{-\lambda \mathrm{T}_{\mathrm{p}}} \mathrm{dT} \mathrm{T}_{\mathrm{p}}+\int_{\mathrm{T}_{\mathrm{p}}=0}^{\mathrm{T}_{1}} \int_{\mathrm{t}=\mathrm{T}_{2}}^{\infty}\left(\mathrm{t}-\mathrm{T}_{2}\right) \mathrm{f}(\mathrm{t}) \lambda \mathrm{e}^{-\lambda \mathrm{T}_{\mathrm{p}}} \mathrm{dT} \mathrm{T}_{\mathrm{p}} \\
& =\frac{\mu\left(\mathrm{ab}^{\mathrm{r}}-1\right)}{\mathrm{ab^{ \textrm {r } }}}+\frac{\ell}{\mathrm{a} \mathrm{b}^{\mathrm{r}}} \frac{\left(1-\mathrm{e}^{-\lambda \mathrm{T}_{1}}\right)}{\lambda}+\frac{\lambda}{2 \mathrm{c}}\left[\frac{\mathrm{A}^{2}\left(1-\mathrm{e}^{-\lambda \mathrm{T}_{1}}\right)}{\lambda}+\frac{\ell^{2}}{\left.(\mathrm{ab})^{\mathrm{r}}\right)^{2}}\left(\frac{-\mathrm{T}_{1}^{2} \mathrm{e}^{-\lambda \mathrm{T}_{1}}}{\lambda}-\frac{2 \mathrm{~T}_{1}}{\lambda^{2}} \mathrm{e}^{-\lambda \mathrm{T}_{1}}+\frac{2}{\lambda^{3}}\left(1-\mathrm{e}^{-\lambda \mathrm{T}_{1}}\right)\right)\right.
\end{aligned}
$$


$\left.-\frac{2 \mathrm{~A} \ell}{\mathrm{ab}^{\mathrm{r}}}\left(\frac{-\mathrm{T}_{1} \mathrm{e}^{-\lambda \mathrm{T}_{1}}}{\lambda}+\frac{1-\mathrm{e}^{-\lambda \mathrm{T}_{1}}}{\lambda^{2}}\right)\right]$

Substituting all the values from Eqs. (5-11), the expected total profit, $E(T P)=E(S R)-E(T C)$ is calculated as follows,

$$
\begin{aligned}
& \left\{\frac{S \alpha \ell\left(e^{((\ell-1) \beta-\lambda) T_{1}}-1\right)}{(\ell-1) \beta-\lambda}+\frac{S \alpha r \lambda e^{-\mu \beta}}{(\ell \beta-\lambda) \beta}-\frac{S \ell \alpha r e^{(\ell \beta-\lambda) T_{1}-\mu \beta}}{(\ell \beta-\lambda)}+\frac{S \alpha r}{\beta}\right\} \\
& -\left\{\mathrm{K}+\mathrm{h}\left(\frac{\alpha \ell \mathrm{e}^{((\ell-1) \beta-\lambda) \mathrm{T}_{1}}}{\beta((\ell-1) \beta-\lambda)}-\frac{\lambda \alpha \ell}{(\ell-1) \beta^{2}((\ell-1) \beta-\lambda)}+\frac{\ell \alpha\left(1-\mathrm{ab}^{\mathrm{r}}\right) \mathrm{e}^{(\ell \beta-\lambda) \mathrm{T}_{1}-\mu \beta}}{\left(\mathrm{ab}^{\mathrm{r}}\right) \beta(\ell \beta-\lambda)}-\frac{\left(1-\mathrm{ab}^{\mathrm{r}}\right) \mathrm{e}^{-\mu \beta} \lambda \alpha}{\left(\mathrm{ab}^{\mathrm{r}}\right) \beta^{2}(\ell \beta-\lambda)}\right)\right\} \\
& +\left\{\frac{\mathrm{h} \alpha}{\beta^{2}}\left(\frac{1}{\ell-1}+\frac{1}{(\mathrm{ab}}\right)+\frac{\mathrm{h} \mu\left(\mathrm{ab}^{\mathrm{r}}-1\right) \alpha}{\left(\mathrm{ab}^{\mathrm{r}}\right) \beta}+\frac{\mathrm{h} \ell \alpha}{\left(\mathrm{ab}^{\mathrm{r}}\right) \beta}\left(\frac{1-\mathrm{e}^{-\lambda \mathrm{T}_{1}}}{\lambda}\right)-\mathrm{C}_{\mathrm{p}} 1 \alpha\left(\frac{\left.\mathrm{e}^{((1-1) \beta-\lambda) \mathrm{T}_{1}}-1\right)}{(1-1) \beta-\lambda}\right)\right\} \\
& -\frac{\lambda \mathrm{S} \alpha}{2 \mathrm{c}}\left\{\frac{\mathrm{A}^{2}\left(1-\mathrm{e}^{-\lambda \mathrm{T}_{1}}\right)}{\lambda}+\frac{\ell^{2}}{\left(\mathrm{a} \mathrm{b}^{\mathrm{r}}\right)^{2}}\left(\frac{-\mathrm{T}_{1}^{2} \mathrm{e}^{-\lambda \mathrm{T}_{1}}}{\lambda}-\frac{2 \mathrm{~T}_{1} \mathrm{e}^{-\lambda \mathrm{T}_{1}}}{\lambda^{2}}+\frac{2\left(1-\mathrm{e}^{-\lambda \mathrm{T}_{1}}\right)}{\lambda^{3}}\right)-\frac{2 \mathrm{~A} \ell}{\mathrm{ab}}\left(\frac{-\mathrm{T}_{1} \mathrm{e}^{-\lambda \mathrm{T}_{1}}}{\lambda}+\frac{1-\mathrm{e}^{-\lambda \mathrm{T}_{1}}}{\lambda^{2}}\right)\right\} \\
& \frac{d E(T P)}{d T_{1}}=S \alpha \ell e^{-\lambda T_{1}}\left(e^{(\ell-1) T_{1} \beta}-r e^{\left(\ell T_{1}-\mu\right) \beta}\right)-\left\{h\left(\frac{\alpha \ell e^{((\ell-1) \beta-\lambda) T_{1}}}{\beta}+\frac{\ell \alpha\left(1-a b^{r}\right) e^{(\ell \beta-\lambda) T_{1}-\mu \beta}}{\left(a b^{r}\right) \beta}-\frac{\ell \alpha e^{-\lambda T_{1}}}{\left(a b^{r}\right) \beta}\right)\right. \\
& \left.+\mathrm{C}_{\mathrm{p}} \ell \alpha \mathrm{e}^{((\ell-1) \beta-\lambda) \mathrm{T}_{1}}+\frac{\lambda \mathrm{S} \alpha}{2 \mathrm{c}}\left(\mathrm{A}^{2} \mathrm{e}^{-\lambda \mathrm{T}_{1}}+\frac{\ell^{2} \mathrm{~T}_{1}^{2} \mathrm{e}^{-\lambda \mathrm{T}_{1}}}{\left(\mathrm{ab}^{\mathrm{r}}\right)^{2}}-\frac{2 \mathrm{~A} \ell \mathrm{T}_{1} \mathrm{e}^{-\lambda \mathrm{T}_{1}}}{\mathrm{ab}}\right)\right\} \\
& \frac{d^{2} E(T P)}{d T_{1}^{2}}=\left\{S \alpha \ell\left((\ell-1) \beta e^{((\ell-1) \beta-\lambda) T_{1}}-r \ell \beta e^{(\ell \beta-\lambda) T_{1}-\mu \beta}\right)\right\}-\left\{h \alpha \ell e^{-\lambda T_{1}}\left((\ell-1) e^{((\ell-1) \beta) T_{1}}+\frac{\ell\left(1-a b^{r}\right) e^{\left(\ell T_{1}-\mu\right) \beta}}{\left(a b^{r}\right)}\right)\right. \\
& \left.C_{p} l(l-1) \beta \alpha e^{((l-1) \beta-\lambda) T_{1}}+\frac{\lambda S \alpha}{c}\left(\frac{\ell^{2} T_{1} e^{-\lambda T_{1}}}{\left(a b^{r}\right)^{2}}-\frac{A \ell e^{-\lambda T_{1}}}{a b^{r}}\right)\right\}
\end{aligned}
$$

Using renewal reward theorem, Expected total average profit, $E(T A P)$

$$
E(T A P)=\frac{E(T P)}{E(T)}=\frac{E(S R)-E(T C)}{E(T)}
$$

\section{Optimal solution procedure:}

Our objective is to determine the expected optimal value of $T_{1}$ so that $E(T A P)$ is maximized. The necessary condition for $E(T A P)$ to be maximized is $\frac{d(E(T A P))}{d T_{1}}=0$ and $\frac{d^{2} E(T A P)}{d T_{1}^{2}}<0$. The expected total profit per unit time is concave where $0 \leq T_{2} \leq c$, (see Appendix A, Appendix B and Appendix C for detailed calculations).

The optimal value of $T_{1}$ has been obtained numerically using Newton Raphson Method.

The following solution procedure is used to derive the optimal values of $T_{1}$ 
We find the root of the equation $\frac{d(E(T A P))}{d T_{1}}=0$ using $C^{++}$program of Newton Raphson Method using the algorithm as below

Step 1: Define $f\left(T_{1}\right)=\frac{d(E(T A P))}{d T_{1}}$

$$
\text { Define } \quad f^{\prime}\left(T_{1}\right)=\frac{d^{2}(E(T A P))}{d T_{1}^{2}}
$$

Step 2: Choose initial value of $T_{1}=t_{0}$ and set values for other parameters.

Step 3: Apply do-while loop

Find $p=\frac{f\left(t_{0}\right)}{f^{\prime}\left(t_{0}\right)}$

Store in $t_{0}=t_{0}-p$

If $|p|<0.0002$, then declare $t_{0}$ to be the required root else repeat Step 3 .

Case II : When $\mu$ is greater than the cycle length

When $\mu$ is greater than the cycle length then there is no sale period and demand remains stock dependent, the governing equations take the form:

$$
\begin{aligned}
& \frac{\mathrm{dq}}{\mathrm{dt}}=(\ell-1)(\alpha+\beta \mathrm{q}), \mathrm{q}(0)=0,0 \leq \mathrm{t} \leq \mathrm{T}_{1} \\
& \frac{\mathrm{dq}}{\mathrm{dt}}=-(\alpha+\beta \mathrm{q}), \mathrm{q}\left(\mathrm{T}_{2}\right)=0, \mathrm{~T}_{1} \leq \mathrm{t} \geq \mathrm{T}_{2}
\end{aligned}
$$

As $\mu \rightarrow T_{1}$, a $\rightarrow 1$ and $\mathrm{r} \rightarrow 0$, in case I, we can have the expected total profit in Case II as $E(T P)=$ $E(S R)-E(T C)$

$$
\begin{gathered}
\left\{\frac{S \alpha \ell\left(e^{((\ell-1) \beta-\lambda) T_{1}}-1\right)}{(\ell-1) \beta-\lambda}\right\}-\left\{\mathrm{K}+\mathrm{h}\left(\frac{\alpha \ell\left(\mathrm{e}^{((\ell-1) \beta-\lambda) \mathrm{T}_{1}}-1\right)}{\beta((\ell-1) \beta-\lambda)}-\frac{\ell \alpha}{\beta}\left(\frac{1-\mathrm{e}^{-\lambda \mathrm{T}_{1}}}{\lambda}\right)\right)+\mathrm{C}_{\mathrm{p}} \ell \alpha\left(\frac{\mathrm{e}^{((\ell-1) \beta-\lambda) \mathrm{T}_{1}}-1}{(\ell-1) \beta-\lambda}\right)\right\} \\
-\frac{\mathrm{s} \alpha}{2 \mathrm{c}}\left\{\mathrm{c}^{2}-\left(\mathrm{c}-\ell \mathrm{T}_{1}\right)^{2} \mathrm{e}^{-\lambda \mathrm{T}_{1}}+\frac{2 \ell}{\lambda}\left(\left(\mathrm{c}-\ell \mathrm{T}_{1}\right) \mathrm{e}^{-\lambda \mathrm{T}_{1}}-\mathrm{c}\right)-\frac{2 \ell^{2}}{\lambda^{2}}\left(\mathrm{e}^{-\lambda \mathrm{T}_{1}}-1\right)\right\}
\end{gathered}
$$

Expected total time, $\mathrm{E}(\mathrm{T})=\mathrm{E}\left(\mathrm{T}_{2}\right)+$ Expected Repair time

$$
=\ell\left(\frac{1-\mathrm{e}^{-\lambda \mathrm{T}_{1}}}{\lambda}\right)+\frac{1}{2 \mathrm{c}}\left\{\mathrm{c}^{2}-\left(\mathrm{c}-\ell \mathrm{T}_{1}\right)^{2} \mathrm{e}^{-\lambda \mathrm{T}_{1}}+\frac{2 \ell}{\lambda}\left(\left(\mathrm{c}-\ell \mathrm{T}_{1}\right) \mathrm{e}^{-\lambda \mathrm{T}_{1}}-\mathrm{c}\right)-\frac{2 \ell^{2}}{\lambda^{2}}\left(\mathrm{e}^{-\lambda \mathrm{T}_{1}}-1\right)\right\}
$$

The expected total profit per unit time is concave where $0 \leq \mathrm{T}_{2} \leq \mathrm{c}$,

\section{Numerical example}

In this part, we have presented computational results obtained by using $\mathrm{C}++$ program of Newton Raphson Method which gives insight about the behavior of expected production cycle time $E(T)$, expected optimal run size $\mathrm{E}(\mathrm{Q})$ and the expected total average profit $E(T A P)$. The parametric values in the models are taken as 
$\beta=0.4, h=2, K=200, \lambda=0.2, \alpha=25, C_{p}=10, S=20, c=5, \mu=3, a=0.5, b=2$

Table 1

Effect of $C_{p}$ on optimal values of $E(Q), E(T)$ and $E(T A P)$

\begin{tabular}{|c|c|c|c|c|}
\hline \multicolumn{5}{|c|}{$\ell=1.8$} \\
\hline$C_{p}$ & 9 & 10 & 11 & 12 \\
\hline$T_{1}$ & 2.652 & 2,444 & 2.2103 & 1.931 \\
\hline $\mathrm{E}(\mathrm{Q})$ & 53.72 & 50.262 & 46.64 & 42.653 \\
\hline $\mathrm{E}(\mathrm{T})$ & 4.679 & 4.3379 & 3.9373 & 3.4305 \\
\hline $\mathrm{E}(\mathrm{HC})$ & 281.131 & 232.846 & 185.957 & 139.670 \\
\hline E(LSC) & 305.612 & 305.375 & 305.267 & 303.678 \\
\hline $\mathrm{E}(\mathrm{PC})$ & 1706.826 & 1849.723 & 1978.426 & 2087.1456 \\
\hline $\mathrm{E}(\mathrm{SR})$ & 3522.820 & 3514.2084 & 3496.94 & 3467.3443 \\
\hline $\mathrm{E}(\mathrm{TAP})$ & 241.34 & 236.581 & 235.514 & 243.944 \\
\hline
\end{tabular}

- As $C_{p}$ increases, the production cost also increases. To balance the high production cost, production run time decreases. Consequently, inventory level also decreases and which leads to decrease in holding cost.

- Higher values of $C_{p}$ leads to lower inventory level. As the demand rate is based on the inventory level and sales revenues are based on the demand, therefore, decrease in inventory level decreases the sales revenues, which also causes fall in the expected total profit.

- As $C_{p}$ increases, expected cycle length, which includes repair time also decreases. It is reasonable that decrease in repair time decreases the lost sales cost also.

Table 2

Effect of $h$ on optimal values of $E(Q), E(T)$ and $E(T A P)$

\begin{tabular}{ccccc}
\hline$h$ & 2.5 & 2 & 1.5 & 1 \\
\hline$C_{p}$ & 1.8142 & 2.3023 & 2.5263 & 2.782 \\
$T_{1}$ & 47.505 & 57.748 & 63.161 & 69.963 \\
$\mathrm{E}(\mathrm{T})$ & 3.6193 & 4.602 & 5.023 & 5.485 \\
$\mathrm{E}(\mathrm{HC})$ & 223.097 & 305.534 & 285.737 & 240.78 \\
$\mathrm{E}(\mathrm{LSC})$ & 277.071 & 277.992 & 278.863 & 282.302 \\
$\mathrm{E}(\mathrm{PC})$ & 1321.98 & 1457.541 & 1524.323 & 1604.305 \\
E(SR) & 2597.82 & 2666.54 & 2684.62 & 2692.99 \\
E(TAP) & 214.315 & 135.83 & 118.595 & 103.119 \\
\hline
\end{tabular}

- As $h$ decreases, the productions run time increases. Rise in production run time raises the production cost.

- Lower values of $h$ leads to higher production run time. Consequently, there is a rise in the inventory level. As the demand rate is based on the inventory level and sales revenues are based on the demand, therefore, increase in inventory level increases the sales revenues. Although there is rise in the sales revenues but total average profit falls in view of rise in some other costs.

- As $h$ decreases, expected cycle length, which includes repair time also increases. It is reasonable that increase in repair time increases the lost sales cost also.

Table 3

Effect of $\ell$ on optimal values of $Q, T$ and $E(T A P)$

\begin{tabular}{cccc}
\hline$\ell$ & 1.6 & 1.8 & 2 \\
\hline$T_{1}$ & 2.609 & 2.444 & 2.3023 \\
$\mathrm{E}(\mathrm{Q})$ & 43.005 & 50.262 & 57.748 \\
$\mathrm{E}(\mathrm{T})$ & 4.0601 & 4.3379 & 4.602 \\
$\mathrm{E}(\mathrm{TAP})$ & 826.639 & 236.581 & 135.8304 \\
\hline
\end{tabular}


- As $\ell$ increases, the production runtime decrease, which is very genuine result to expect. Although in view of the increase in production rate there is increase in the inventory level.

- As production rate increases, there is increase in the cycle length.

- Higher values of $\ell$ leads to lower expected total average profit.

- Higher production run time and lower production rate give higher profit.

\section{Conclusion}

The model developed above addressed some expected realistic features that usually arose while working on the optimal production policy for stochastic models that maximized the expected profit. It was very important to take the production system as unreliable as uncertainty was very expected feature of a real system. If machine broke down, it was always not certain that it could be repaired in a fixed time period. Normally, in the supermarkets the demand was influenced by the stock displayed on their shelves. In view of the highly competitive situation in the real business, the production system could not afford to be inflexible. Keeping in view all the issues raised, we took the production system unreliable and flexible, taking production rate as a function of demand. In addition, we took a machine repair time stochastic and derived an optimal production policy for the stochastic model which could maximize the expected profit. It was observed that increase in the production rate decreased the production run time and decrease in the production rate increased the production run time. Further, it was observed that longer production up time and lower production rates provided higher expected profits as compared to shorter production uptime and higher production rates in stochastic model. It was also observed that the higher production cost per unit decreased the expected profit. Further research on the problem could be extended to consider more realistic assumptions into the proposed model, for example, imperfect quality of products, reverse manufacturing, trade credit, etc.

\section{References}

Chakraborty, T., Giri, B. C., \& Chaudhuri, K. S. (2008). Production lot sizing with process deterioration and machine breakdown. European Journal of Operational Research, 185(2), 606-618.

Cheung, K. L., \& Hausman, W. H. (1997). Joint determination of preventive maintenance and safety stocks in an unreliable production environment. Naval Research Logistics (NRL), 44(3), 257-272.

Chiu, S. W., Wang, S. L., \& Chiu, Y. S. P. (2007). Determining the optimal run time for EPQ model with scrap, rework, and stochastic breakdowns. European Journal of Operational Research, 180(2), 664-676.

Dem, H., \& Singh, S. R. (2012, January). Production scheduling for damageable items with demand and cost flexibility using genetic algorithm. In Proceedings of the International Conference on Soft Computing for Problem Solving (SocProS 2011) December 20-22, 2011 (pp. 747-759). Springer India.

Dem, H., \& Singh, S. R. (2013). A production model for ameliorating items with quality consideration. International Journal of Operational Research, 17(2), 183-198.

El-Ferik, S. (2008). Economic production lot-sizing for an unreliable machine under imperfect agebased maintenance policy. European Journal of Operational Research, 186(1), 150-163.

Giri, B. C., \& Chaudhuri, K. S. (1998). Deterministic models of perishable inventory with stockdependent demand rate and nonlinear holding cost. European Journal of Operational Research, 105(3), 467-474.

Giri, B. C., Yun, W. Y., \& Dohi, T. (2005). Optimal design of unreliable production-inventory systems with variable production rate. European Journal of Operational Research, 162(2), 372-386.

Goyal, S. K., Singh, S. R., \& Dem, H. (2013). Production policy for ameliorating/deteriorating items with ramp type demand. International Journal of Procurement Management, 6(4), 444-465.

Groenevelt, H., Pintelon, L., \& Seidmann, A. (1992). Production lot sizing with machine breakdowns. Management Science, 38(1), 104-123. 
Groenevelt, H., Pintelon, L., \& Seidmann, A. (1992). Production batching with machine breakdowns and safety stocks. Operations Research, 40(5), 959-971.

Gupta, R., \& Vrat, P. (1986). Inventory model for stock-dependent consumption rate. Opsearch, 23(1), 19-24.

Konstantaras, I., \& Skouri, K. (2010). Lot sizing for a single product recovery system with variable setup numbers. European Journal of Operational Research, 203(2), 326-335.

Levin, R. I., Mclaughlin, C. P., Lamone, R. P., \& Kottas, J. F. (1972). Production, Operations Management: Contemporary Policy for Managing Operation System. NewYork: McGraw-Hill.

Leung, K. N. F. (2007). A generalized geometric-programming solution to "An economic production quantity model with flexibility and reliability considerations". European Journal of Operational Research, 176(1), 240-251.

Liao, G. L., Chen, Y. H., \& Sheu, S. H. (2009). Optimal economic production quantity policy for imperfect process with imperfect repair and maintenance.European Journal of Operational Research, 195(2), 348-357.

Lin, G.C., \& Gong, D.C. (2006). On a production inventory system of deteriorating items subject to random machine breakdowns with a fixed repair time. Mathematical Computational Modeling, 43(7), 920-932.

Mandal, B.N., \& Phaujdar, S. (1989). An inventory model for deteriorating items and stock dependent consumption rate. Journal Operational Research Society, 40, 483-488.

Peterson, R., \& Silver, E. A. (1979). Decision systems for inventory management and production planning (pp. 799-799). New York: Wiley.

Sana, S., Goyal, S.K., \& Chaudhuri K.S. (2006). An imperfect production process in a volume flexible inventory model. International Journal of Production Economics, 105,548-559.

Schweitzer, P. J., Seidmann, A. (1991). Optimizing, processing rate for flexible manufacturing systems. Management Science, 37, 454-466.

Singh, S. R., Kumar, N., \& Kumari, R. (2010). An inventory model for deteriorating items with shortages and stock-dependent demand under inflation for two-shops under one management. Opsearch, 47(4), 311-329.

Singh, S. R., \& Saxena, N. (2012). An optimal returned policy for a reverse logistics inventory model with backorders. Advances in Decision Sciences.

Singh, S. R., \& Jain, R. (2009). On reserve money for an EOQ model in an inflationary environment under supplier credits. Opsearch, 46(3), 303-320.

Singh, S. R., \& Singh, C. (2010). Supply chain model with stochastic lead time under imprecise partially backlogging and fuzzy ramp-type demand for expiring items. International Journal of Operational Research, 8(4), 511-522.

Teng, J. T., \& Chang, C. T. (2005). Economic production quantity models for deteriorating items with price-and stock-dependent demand. Computers \& Operations Research, 32(2), 297-308.

Wang, C. H. (2004). The impact of a free-repair warranty policy on EMQ model for imperfect production systems. Computers \& Operations Research, 31(12), 2021-2035.

Widyadana, G. A., \& Wee, H. M. (2011). Optimal deteriorating items production inventory models with random machine breakdown and stochastic repair time. Applied Mathematical Modelling, 35(7), 3495-3508.

Yadav, D., Singh, S. R., \& Kumari, R. (2012). Inventory model of deteriorating items with twowarehouse and stock dependent demand using genetic algorithm in fuzzy environment. Yugoslav Journal of Operations Research ISSN: 0354-0243 EISSN: 2334-6043, 22(1). 


\section{Appendix A:}

\section{Derivative analysis of expected time function:}

For feasibility of the practical situation considered in the model $T_{2}$ must be less than c, otherwise there will be no lost sales interval as lost sales occur when repair time exceeds $T_{2}$. So we consider the behavior of $\mathrm{E}(\mathrm{T})$ in interval $0 \leq T_{2} \leq c$

$$
\frac{\mathrm{dE}(\mathrm{T})}{\mathrm{dT}_{1}}=\left(\frac{\ell}{\mathrm{ab}^{\mathrm{r}}}+\frac{\lambda \mathrm{A}^{2}}{2 \mathrm{c}}+\frac{\ell^{2} \lambda}{2 \mathrm{c}\left(\mathrm{ab}^{\mathrm{r}}\right)^{2}} \mathrm{~T}_{1}^{2}-\frac{2 \mathrm{~A} \ell \lambda \mathrm{T}_{1}}{\left(\mathrm{ab} \mathrm{b}^{\mathrm{r}}\right) 2 \mathrm{c}}\right) \mathrm{e}^{-\lambda \mathrm{T}_{1}} \frac{\mathrm{d}^{2} \mathrm{E}(\mathrm{T})}{\mathrm{dT}_{1}^{2}}=\mathrm{e}^{-\lambda \mathrm{T}_{1}}\left(\frac{\ell^{2} \lambda \mathrm{T}_{1}}{\left(\mathrm{ab} \mathrm{b}^{\mathrm{r}}\right)^{2} \mathrm{c}}-\frac{\mathrm{A} \ell \lambda}{\left(\mathrm{ab} \mathrm{b}^{\mathrm{r}}\right) \mathrm{c}}\right)
$$

Set $\mathrm{T}_{1}=0$, we have $\frac{\mathrm{d}^{2} \mathrm{E}(\mathrm{T})}{\mathrm{dT}_{1}^{2}}=-\frac{\mathrm{A} \ell \lambda}{\left(a b^{\mathrm{r}}\right) \mathrm{c}}<0$

$\frac{\mathrm{d}^{2} \mathrm{E}(\mathrm{T})}{\mathrm{dT}_{1}^{2}}<0$ If $\frac{\ell^{2} \lambda}{\left(\mathrm{ab}^{\mathrm{r}}\right)^{2} \mathrm{c}} \mathrm{T}_{1}<\frac{\mathrm{A} \ell \lambda}{\left(\mathrm{ab}^{\mathrm{r}}\right) \mathrm{c}} \Rightarrow \mathrm{T}_{1}<\frac{\mathrm{A}\left(\mathrm{ab}^{\mathrm{r}}\right)}{\ell}$

$E(T)$ is concave w.r.t. $T_{1}$, where $0 \leq \mathrm{T}_{1} \leq \frac{\mathrm{A}\left(\mathrm{ab}^{\mathrm{r}}\right)}{\ell}$, where $\mathrm{A}=\frac{\mathrm{c}\left(\mathrm{ab}^{\mathrm{r}}\right)-\mu\left(\mathrm{ab}^{\mathrm{r}}-1\right)}{\mathrm{ab}^{\mathrm{r}}}$

Also $0 \leq \mathrm{T}_{1} \leq \frac{\mathrm{A}\left(\mathrm{ab}^{\mathrm{r}}\right)}{\ell} \Rightarrow 0 \leq T_{2} \leq c$

Therefore, $E(T)$ is concave when $0 \leq T_{2} \leq c$

\section{Appendix B:}

To prove, $\frac{d^{2} E(T A P)}{d T_{1}^{2}}<0$ if $\frac{d^{2} E(T P)}{d T_{1}^{2}}<0$ where $0 \leq T_{2} \leq c$

$\frac{d^{2} E(T A P)}{d T_{1}^{2}}=\frac{d^{2}\left(\frac{E(T P)}{E(T)}\right)}{d T_{1}^{2}}=\frac{E(T) \frac{d^{2} E(T P)}{d T_{1}^{2}}-E(T P) \frac{d^{2} E(T)}{d T_{1}^{2}}}{(E(T))^{2}}$

Now $\frac{d^{2} E(T A P)}{d T_{1}^{2}}<0$ if $E(T) \frac{d^{2} E(T P)}{d T_{1}^{2}}<E(T P) \frac{d^{2} E(T)}{d T_{1}^{2}}$

Using (A1), As $\frac{d^{2} E(T)}{d T_{1}^{2}}<0$ in the interval $0 \leq T_{2} \leq c$

Also $\mathrm{E}(T)>0, \mathrm{E}(T P)>0$,

Therefore, in the interval $0 \leq T_{2} \leq c, \frac{d^{2} E(T A P)}{d T_{1}^{2}}<0$ if $\frac{d^{2} E(T P)}{d T_{1}^{2}}<0$

\section{Appendix C:}

To prove $\frac{d^{2} E(T P)}{d T_{1}^{2}}<0$

$$
\begin{aligned}
& \frac{d(E(S R))}{d T_{1}}=S \alpha \ell e^{-\lambda T_{1}}\left(e^{(\ell-1) \beta}-r e^{\left(\ell T_{1}-\mu\right) \beta}\right) \\
& \frac{d^{2}(E(S R))}{d T_{1}^{2}}=S \alpha \ell e^{-\lambda T_{1}}\left((\ell-1) \beta e^{(\ell-1) \beta T_{1}}-r \ell \beta e^{\left(\ell T_{1}-\mu\right) \beta}\right)-\lambda \frac{d E(S R)}{d T_{1}}
\end{aligned}
$$




$$
\begin{aligned}
& (\ell-1) \beta e^{(\ell-1) \beta T_{1}}-r \ell \beta e^{\left(\ell T_{1}-\mu\right) \beta}<0 \text { If } \frac{(\ell-1)}{r \ell}<\frac{e^{(\ell-1) \beta T_{1}}}{e^{\left(\ell T_{1}-\mu\right) \beta}} \\
& \Rightarrow \text { If } \frac{(\ell-1)}{r \ell}<e^{-\left(\mu-T_{1}\right) \beta T_{1}}<\min \left(e^{-\left(\mu-T_{1}\right) \beta T_{1}}\right)=1
\end{aligned}
$$

AS $l>1 \Rightarrow \frac{1}{l}<1$

Therefore $\frac{d^{2}(E(S R))}{d T_{1}^{2}}<0$ if $(1-r)<1$ which holds true

$$
\begin{aligned}
& \frac{d(E(T C))}{d T_{1}}= \\
& \left.\left\{h\left(\frac{\alpha \ell e^{((\ell-1) \beta-\lambda) T_{1}}}{\beta}+\frac{\ell \alpha\left(1-a b^{r}\right) e^{(\ell \beta-\lambda) T_{1}-\mu \beta}}{\left(a b^{r}\right) \beta}-\frac{\ell \alpha e^{-\lambda T_{1}}}{\left(a b^{r}\right) \beta}\right)+\frac{\lambda S \alpha}{2 c}\right) A^{2} e^{-\lambda T_{1}}+\frac{\ell^{2} T_{1}^{2} e^{-\lambda T_{1}}}{\left(a b^{r}\right)^{2}}-\frac{2 A \ell T_{1} e^{-\lambda T_{1}}}{a b^{r}}\right) \\
& \left.+\mathrm{C}_{\mathrm{p}}\left(\ell \alpha \mathrm{e}^{((\ell-1) \beta-\lambda) \mathrm{T}_{1}}\right)+\frac{\lambda \mathrm{S} \alpha}{2 \mathrm{c}}\left(\mathrm{A}^{2} \mathrm{e}^{-\lambda \mathrm{T}_{1}}+\frac{\ell^{2} \mathrm{~T}_{1}^{2} \mathrm{e}^{-\lambda \mathrm{T}_{1}}}{\left(\mathrm{ab}^{\mathrm{r}}\right)^{2}}-\frac{2 \mathrm{~A} \ell \mathrm{T}_{1} \mathrm{e}^{-\lambda T_{1}}}{a b^{\mathrm{r}}}\right)\right\} \\
& \frac{d^{2}(E(T C))}{d T_{1}^{2}}=\left[h \alpha \ell e^{-\lambda T_{1}}\left((\ell-1) e^{((\ell-1) \beta) T_{1}}+\frac{\ell\left(1-a b^{r}\right) e^{\left(\ell T_{1}-\mu\right) \beta}}{\left(a b^{r}\right)}\right)+\frac{\lambda S \alpha}{c}\left(\frac{\ell^{2} T_{1} e^{-\lambda T_{1}}}{\left(a b^{r}\right)^{2}}-\frac{A \ell e^{-\lambda T_{1}}}{a b^{r}}\right)\right] \\
& +C_{p} l(l-1) \beta \alpha e^{((l-1) \beta-\lambda) T_{1}} \quad-\lambda \frac{d(E(T C))}{d T_{1}}
\end{aligned}
$$

Set $T_{1}=0$

$$
\frac{d^{2}(E(T C))}{d T_{1}^{2}}>0 \text { If }\left(h \alpha \ell\left((\ell-1)+\frac{\ell\left(1-a b^{r}\right) e^{-\mu \beta}}{\left(a b^{r}\right)}\right)-\frac{\lambda S \alpha A \ell}{c a b^{r}}+C_{p} l(l-1) \beta \alpha\right)>0
$$

Set $T_{1}=\frac{A\left(a b^{r}\right)}{\ell}$

$$
\frac{d^{2}(E(T C))}{d T_{1}^{2}}>0 \text { If } h \alpha \ell\left((\ell-1)+\frac{\ell\left(1-a b^{r}\right) e^{-\mu \beta}}{\left(a b^{r}\right)}\right)+C_{p} l(l-1) \beta \alpha e^{((l-1) \beta-\lambda) T_{1}}>0
$$

Now $\frac{d^{2}(E(T P))}{d T_{1}^{2}}=\frac{d^{2}(E(S R))}{d T_{1}^{2}}-\frac{d^{2}(E(T C))}{d T_{1}^{2}}$

Using (C1), (C2), and (C3), we have $\frac{d^{2}(E(S R))}{d T_{1}^{2}}<0$ and $\frac{d^{2}(E(T C))}{d T_{1}^{2}}>0$ in the interval $0 \leq T_{2} \leq c$ when (C2) and (C3) are fulfilled.

Therefore, $\frac{d^{2}(E(T P))}{d T_{1}^{2}}<0$ which in turn implies $\frac{d^{2} E(T A P)}{d T_{1}^{2}}<0$ where $0 \leq T_{2} \leq c$ 